\title{
A fundamental distinction in early neural processing of implicit social interpretation in schizophrenia and bipolar disorder
}

\author{
Nuno Madeira ${ }^{\text {a,b,c,1 }}$, Ricardo Martins ${ }^{\text {a,d,1 }}$, João Valente Duarte ${ }^{\text {a,b,d }}$, Gabriel Costa ${ }^{\text {a,d }}$, \\ António Macedo $^{\text {a,b,c,2, Miguel Castelo-Branco }}{ }^{\text {a,b, d,2,"* }}$

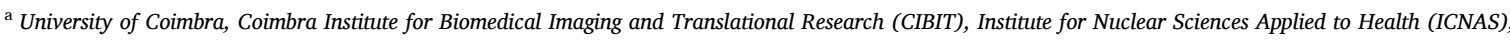 \\ Portugal \\ ${ }^{\mathrm{b}}$ University of Coimbra, Faculty of Medicine (FMUC) - Institute of Psychological Medicine, Portugal \\ ${ }^{\mathrm{c}}$ Centro Hospitalar e Universitário de Coimbra (CHUC) - Department of Psychiatry, Portugal \\ ${ }^{\mathrm{d}}$ University of Coimbra, Faculty of Medicine (FMUC), Portugal
}

\section{A R T I C L E I N F O}

\section{Keywords:}

Social cognition

Theory of mind

Bipolar disorder

Schizophrenia

Temporoparietal junction

Functional magnetic resonance imaging

\begin{abstract}
A B S T R A C T
Background: Social cognition impairment is a key phenomenon in serious mental disorders such as schizophrenia (SCZ) and bipolar disorder (BPD). Although genetic and neurobiological studies have suggested common neural correlates, here we hypothesized that a fundamental dissociation of social processing occurs at an early level in these conditions.

Methods: Based on the hypothesis that key structures in the social brain, namely the temporoparietal junction, should present distinctive features in SCZ and BPD during low-level social judgment, we conducted a case-control study in SCZ $(n=20)$ and BPD $(n=20)$ patients and controls $(n=20)$, using task-based fMRI during a Theory of Mind (ToM) visual paradigm leading to interpretation of social meaning based on simple geometric figures.

Results: We found opposite neural responses in two core ToM regions: SCZ patients showed social content-related deactivation (relative to controls and BPD) of the right supramarginal gyrus, while the opposite pattern was found in BPD; reverse patterns, relative to controls and SCZ, were found in the left posterior superior temporal gyrus, a region involved in inferring other's intentions. Receiver-operating-characteristic curve analysis showed $88 \%$ accuracy in discriminating the two clinical groups based on these neural responses.

Conclusions: These contrasting activation patterns of the temporoparietal junction in SCZ and BPD represent mechanistic differences of social cognitive dysfunction that may be explored as biomarkers or therapeutic targets.
\end{abstract}

\section{Introduction}

An individual can generate cognitive representations of his relations with others and use them flexibly to guide interpersonal behaviors. This ability to recognize, handle and act accordingly with socially relevant information describes social cognition (SC) (Adolphs, 2001). SC dysfunction, manifested by erroneous intention interpretations or idiosyncratic reactions to others' emotions, has profound impact on functioning and daily life (Kern and Horan, 2010). Neurocognitive processes of SC can be systematized in four main areas: emotion processing, social perception, attributional style and theory of mind (ToM) (Green and
Horan, 2010).

SC dysfunction, namely of ToM - the ability to infer others' intentions, emotions and beliefs (Premack and Woodruff, 1978), has been described in severe mental disorders such as schizophrenia (SCZ), representing its most important predictor of global functioning (Couture et al., 2006; Roberts and Penn, 2013). SC has also been implicated as a key contributor to functioning in bipolar disorder (BPD) patients, and deficits were linked with psychosocial disadvantage (Roberts and Penn, 2013; Lahera, 2012; Cusi, 2012). Genetic studies have shown a complex polygenic picture for the architecture of psychiatric disorders (Rees and Owen, 2020). Several common genetic variants were identified (Cross-

\footnotetext{
* Corresponding author at: Coimbra Institute for Biomedical Imaging and Translational Research (CIBIT), Portugal, ICNAS, Universidade de Coimbra, Azinhaga Santa Comba, Celas, 3000-548 Coimbra, Portugal.

1 These authors contributed equally to this work.

2 These authors contributed equally to this work.
} 
Disorder Group of the Psychiatric Genomics, C, 2013) that confer risk to both SCZ and BPD, as well as to autism spectrum disorders - the archetype of SC dysfunction - and current evidence favors a differentiation between SCZ and BPD that is more dimensional than categorical (Craddock et al., 2009). However, to our knowledge, no study has so far focused on the critical distinctions between neural processing of SC in these conditions.

The neural basis of SC involves a complex network of brain areas: ventromedial and dorsolateral prefrontal cortex (PFC), anterior cingulate cortex (ACC), amygdala, temporoparietal junction (TPJ) and insula (Cusi, 2012; Adolphs, 2009). Despite some heterogeneity in basic and clinical studies of SC neural correlates, two meta-analyses of functional neuroimaging studies using ToM paradigms have consistently identified the TPJ and medial PFC as core areas (Schurz, 2014; Van Overwalle, 2009). After initial evidence for the distinction between implicit and explicit understanding in ToM (Clements and Perner, 1994), a two-path ToM system (Apperly and Butterfill, 2009; Butterfill and Apperly, 2013) has been supported, accounting for the developmental dissociation of mentalizing capacity: (1) implicit ToM, arising in early development (first 2 years of life), allowing spontaneous mental-state tracking and rapid extraction of others' mental states; (2) later developing explicit ToM, with conscious analyses of external mental states, supported on additional executive functions (Naughtin, 2017; Filmer et al., 2019). Of ToM's core regions, only the TPJ has been shown to contribute in explicit and implicit processing, as early as in 7-month-old infants (Filmer et al., 2019; Hyde, 2018). This area, namely the right TPJ, has been proposed as a cluster of subregions: anterior TPJ, posterior TPJ and inferior parietal lobe regions (Bzdok, 2013; Mars, 2012). The TPJ and posterior superior temporal sulcus (pSTS) - the TPJ-pSTS complex, a phylogenetically recent hub, coordinate multiple brain networks when exploring dynamic social scenes typical of human experience (Patel et al., 2019).

ToM's neural basis has been studied in SCZ patients. Despite literature inconsistencies, a meta-analysis of $21 \mathrm{fMRI}$ trials with ToM paradigms in SCZ reported hypoactivity of its core network, with abnormal hyperactivation of attentional networks, suggesting vicarious mechanisms to ameliorate behavioral performance, which is frequently below par (Green et al., 2015; Kronbichler, 2017). For BPD, there is limited data on functional neuroimaging about ToM; a relative general hypoactivation of SC key areas has been suggested (Kim, 2009; Malhi, 2008; Willert, 2015). However, direct comparison of SC's specific neural functional correlates in both SCZ and BPD is currently missing. Moreover, a neurobiological distinction of social cognition processing in these conditions remains to be identified, acknowledging that social cognitive dysfunction might not serve as a promising endophenotype in BPD, unlike in SCZ, being more state than trait-related, in addition to its intermediate severity (Lee, 2013). Considering that SCZ has been modelled as a (late) neurodevelopmental disorder, with specific early arising brain changes being explored as biomarkers of this condition (Madeira, 2020; Rapoport et al., 2012), we hypothesized that key social brain structures, involved in early emerging processes, namely the TPJ, could present distinctive features in SCZ vs. euthymic BPD during tasks that require implicit, low-level, social judgment. To test this hypothesis, we investigated the neural substrates of ToM in patients with SCZ and $\mathrm{BPD}$, using a ToM task requiring social interpretation of simple geometric figures.

\section{Methods and materials}

\subsection{Participants}

Right-handed patients with BPD $(\mathrm{n}=20)$ and SCZ $(\mathrm{n}=20)$ matched for age (18-54), gender and education were recruited from the outpatient setting of a large tertiary hospital. Handedness was assessed through the Edinburgh Handedness Inventory (Espírito-Santo, 2017). All patients had an ICD-10 diagnosis of BPD or SCZ confirmed through direct interview by an experienced psychiatrist and medical records reviewing. Clinical stability as an inclusion criteria was operationalized through unchanged medication for at least 3 months, with a similar period of clinical stability: sustained euthymia (Brief Psychiatric Rating Scale's - BPRS (de Almeida, et al., 1996) mania and depression items $\leq$ 1) in BPD patients, and, in SCZ, Positive and Negative Symptoms Scale for Schizophrenia - PANSS (Kay et al., 1987) - score variation under $10 \%$. Exclusion criteria included medical or neurological conditions (e. g. epilepsy, head trauma), comorbid alcohol or other drugs abuse/ dependence, and MRI contraindications. The study was approved by the Ethics Commission of the Faculty of Medicine of the University of Coimbra (ref. CE-010/2014), conducted in accordance with the Declaration of Helsinki. All participants provided written informed consent to participate. Twenty right-handed healthy controls matched for age, gender and education were recruited from hospital and faculty workers and their relatives; only subjects with no personal or first-degree family history of psychiatric disorders were included.

\subsection{Neuropsychological assessments}

Patients were assessed with the Insight and Treatment Attitudes Questionnaire (ITAQ) (Almeida et al., 2005) and Personal and Social Performance Scale (PSP) (Brissos, 2012), measuring, respectively, insight and social functioning. Patients and controls underwent neuropsychological assessment using the following SC psychometric instruments: Face Emotion Identification Test (FEIT) (Kerr and Neale, 1993); Social Perception Scale (SPS) (Garcia, 2003); Schema Component Sequencing Task - Revised (SCST) (Corrigan and Addis, 1995); Ambiguous Intentions Hostility Questionnaire (AIHQ) (Combs, 2007); Reading the Mind in the Eyes Test (RMET) (Baron-Cohen, 2001); Mentalization Questionnaire (MZQ) (Hausberg, 2012); Toronto Empathy Questionnaire (TEQ) (Spreng, 2009). We used the vocabulary subtest of the Wechsler Adult Intelligence Scale - WAIS-III (Wechsler, 2008) as an estimate of premorbid intelligence (Fuentes-Durá, 2019; Bright and van der Linde, 2020). Evaluation was conducted within a 2-week interval after fMRI assessment.

\subsection{Stimuli Presentation and apparatus}

The experimental sessions were designed in Presentation software (version 17.0, Neurobehavioral Systems Inc., Albany, USA) and shown inside the MRI scanner bore by means of an LCD screen (NNL LCD Monitor, NordicNeuroLab, Bergen, Norway; resolution $1920 \times 1080$, refresh rate $60 \mathrm{~Hz}$ ) located approximately $156 \mathrm{~cm}$ away. Participants viewed the screen through a mirror mounted above their eyes. Behavioral responses were collected using two fiber-optical MRI-compatible response pads, Lumina LS-PAIR (Cedrus Lumina LP-400, LU400 PAIR; Cedrus Corporation, San Pedro, USA), held in both hands.

\subsection{Experimental design and procedure}

Participants were engaged in a visual task involving ToM animated stimuli (Tavares et al., 2008; Tavares et al., 2011). An experimental run consisted of 8 trials, each comprising a sequence of baseline, animation movie, jittering, and question blocks. The animation movie block, presented during $14 \mathrm{~s}$, displayed simple geometric shapes (two circles) on a bidimensional structured scenario (animations available as supplementary material). Prior to fMRI sessions, participants were told to interpret the animations as representing social interactions between two agents. There were four different animation categories: affiliative (friendly social interaction), antagonistic (hostile social interaction), indifferent (non-interacting social movements), linear (rectilinear nonsocial movements). Animations categories were presented twice per run, and animation blocks were preceded by a baseline block displayed for $12.5-13.5 \mathrm{~s}$, followed by a short jittering block (0-1 s). Both blocks displayed a fixation circle. The variable duration of baseline and 
jittering blocks was used to ensure that fMRI volumes would not be acquired always in the same temporal instant of the different trials, so as to capture a more complete sampling of the hemodynamic response (Amaro and Barker, 2006). During question blocks an interrogative sentence was presented for $8.5 \mathrm{~s}$, asking participants to classify the emotional valence of social behaviors shown in preceding animations as positive (affiliative), negative (antagonistic), or indifferent (indifferent or linear animation). Participants were instructed to use the right index, right middle finger, and left index to indicate a positive, negative, or indifferent emotional valence, respectively. There were three different sets of randomized runs. Trios of participants (control, SCZ, BPD), matched during recruitment process, performed the same set of 4 randomized runs. Two SCZ participants performed two and three experimental runs, respectively, due to fatigue.

\section{5. fMRI data acquisition and Pre-processing}

Data were collected with a Siemens Magnetom TIM Trio 3T scanner (Siemens, Munich, Germany) with a phased array 12-channel birdcage head coil. The MRI scanning session began by acquiring a 3-D anatomical T1-weighted MPRAGE (magnetization-prepared rapid gradient echo) pulse sequence ( $\mathrm{TR}=2530 \mathrm{~ms}$; $\mathrm{TE}=3.42 \mathrm{~ms}$; $\mathrm{TI}=1100 \mathrm{~ms}$; flip angle $7^{\circ} ; 176$ single-shot interleaved slices [no inter-slice gap] with voxel size 1x1x1mm; FOV $256 \mathrm{~mm}$ ). Functional images were acquired axially using a $\mathrm{T} 2 \%$-weighted gradient echo (GE) echo planar imaging (EPI) sequence covering the whole brain. Each functional series consisted of 150 volumes (TR $=2000 \mathrm{~ms}, \mathrm{TE}=39 \mathrm{~ms}$, flip angle $=90^{\circ}, 29$ interleaved slices [no inter-slice gap] with voxel size $3 \times 3 \times 4 \mathrm{~mm}$; FOV $254 \mathrm{~mm}$ ) of BOLD signal measurements.

\section{6. $f M R I$ data analysis}

Data processing was performed using BrainVoyager 21.2 (Brain Innovation, The Netherlands). Functional volumes pre-processing included slice-scanning time correction, interscan 3D head-motion correction, temporal high-pass filtering (GLM approach with a Fourier basis set of 2 cycles, which removes low frequencies equal or below to $0.006 \mathrm{~Hz}$ ) and linear-trend removal. Functional data were normalized to Talairach anatomical space and spatially smoothed with a Gaussian kernel of 6-mm full-width at half-maximum.

For each experimental run, a general linear model (GLM) design matrix was defined, with box-car predictors for each experimental condition and confound predictors from head motion parameters and spikes. Predictors were convolved with the canonical hemodynamic response function (HRF). Then, resulting single-run beta values entered a second-level group random effects (RFX) GLM analysis.

\subsection{Statistical data analysis}

Differences in age and education levels were assessed in patients (BPD or SCZ) and healthy participants through ANOVA. Independent samples $t$-test or chi-square comparisons of clinical data between BPD and SCZ patients were conducted.

Two behavioral measures of performance on the fMRI task were calculated: correct expected response percent score and average time spent to report a decision during the question block. For these variables, the effects of group and video animation category were assessed independently using Independent Samples Kruskal-Wallis $\mathrm{H}$ tests and Friedman tests, respectively.

Demographic, clinical and fMRI task behavior statistical analyses were performed using SPSS 24 (IBM, Armonk, USA).

To test our hypothesis of differential activation of the neural substrates underlying low-level ToM processing, a region of interest (ROI) was defined using a large bilateral TPJ mask in Talairach space. This extensive mask, with 25,083 voxels, comprising a comprehensive set of ToM brain regions, was obtained by mirroring and converting to
Talairach space (Lacadie, 2008) an existing right TPJ mask in MNI space (Mars, 2012). A ROI voxel-wise RFX-GLM group analysis was conducted using functional data from all experimental runs. Resulting beta values were used to perform a voxel-wise two-way mixed ANOVA analysis with one within-subjects factor with repeated measures (category of animation video: affiliative, antagonistic, indifferent, linear) and one betweensubjects factor (group: CTR, SCZ, BPD). The voxel-wise two-way mixed ANOVA was used to test between-subjects' differences, and the interaction between animation video category and group. Group level statistical F-maps were corrected for multiple comparisons using false discovery rate (FDR) method at $\mathrm{q}(\mathrm{FDR})<0.05$.

In addition, a ROI RFX-GLM analysis was conducted within each cluster presenting a statistically significant interaction of category vs. group, to extract a single beta value for each predictor per subject. The ROI RFX-GLM beta values were computed and used to study, using SPSS 24 , the simple main-effect of the factor group for the affiliative, antagonistic, indifferent, and linear animation videos.

The receiver-operating-characteristic (ROC) analysis (sensitivity vs 1-specificity) was carried on the ROI RFX-GLM beta values for each pairing of groups following univariate and multivariate classification approaches. The area under the ROC curve (AUC) was used as performance metric. The univariate approach, performed using SPSS 24, applied the ROC analysis independently to the beta values of the affiliative, antagonistic, indifferent, and linear predictors. The multivariate approach, implemented using MATLAB R2019 (MathWorks Inc., USA) and the Statistics and Machine Learning Toolbox, applied ROC analysis to the classification scores determined by binary linear support vector machine classifiers (10-fold cross validation, 50 runs) designed with four features corresponding to the beta values of the affiliative, antagonistic, indifferent, and linear predictors.

BOLD signal event-related average time courses were computed as follows: in each trial of the different animation video categories, percent BOLD signal change was determined using the start of the video as trigger, and as baseline the preceding period of 2 volumes (4 $\mathrm{s}$ of baseline block, displaying a fixation circle). Segments representing the same category of animation video were averaged over runs for the different groups of participants: controls, SCZ and BPD.

\section{Results}

Demographic and clinical data regarding participants can be found in Table 1.

\subsection{Psychopathology}

Patients from both clinical groups were either in remission or sustained clinical stability, as shown by mean (SD) BPRS scores for patients with BPD [29.1(2.6)] and SCZ [35.6(6.4)]. Differences between two groups were statistically significant $(\mathrm{p}<.001)$. PANSS mean score in the SCZ sample was 44.7(SD = 9.6). BPD patients had better social functioning compared to SCZ [PSP scores, 92.0(4.0)] vs 80.2(12.4), p = .001]. Insight was also lower in the SCZ sample: ITAQ mean of 17.1(3.2) vs. 19.1(2.2) in BPD patients.

\subsection{Cognition}

In general, clinical groups had lower scores in SC measures, but differences were not significant compared to controls in facial emotion identification (FEIT), attributive style (AIHQ) and in the Reading-theMind-in-the-Eyes-Test (RMET).

The SCZ group had significantly lower performance than controls in social schemas assessment (SCST, $\mathrm{p}=0.015$ ), empathy (TEQ, $\mathrm{p}=$ 0.009 ), and emotional awareness (MZQ-EA subscale, $p=0.002$ ). Differences in social perception (SPS, $\mathrm{p}<0.001$ ) between patients and controls (49.25 \pm 6.90 ) were particularly evident, with intermediate dysfunction found in BPD (39.90 \pm 6.09$)$, and more severe impairment 
Table 1

Demographic and psychopathologic sample characteristics.

\begin{tabular}{|c|c|c|c|c|c|}
\hline \multirow[b]{2}{*}{$\begin{array}{l}\text { Gender, male/ } \\
\text { female }\end{array}$} & \multirow{2}{*}{$\begin{array}{l}\text { Schizophrenia } \\
13 / 7\end{array}$} & \multirow{2}{*}{$\begin{array}{l}\begin{array}{l}\text { Bipolar } \\
\text { Disorder }\end{array} \\
13 / 7\end{array}$} & \multirow{2}{*}{$\begin{array}{l}\text { Controls } \\
13 / 7\end{array}$} & \multicolumn{2}{|c|}{ Test statistics } \\
\hline & & & & N/A & N/A \\
\hline $\begin{array}{l}\text { Age, years } \\
\quad(\text { mean } \pm \mathrm{SD})\end{array}$ & $31.5 \pm 10.3$ & $31.6 \pm 10.0$ & $\begin{array}{l}31.5 \pm \\
10,3\end{array}$ & $\begin{array}{l}F= \\
0.001^{(a)}\end{array}$ & $\begin{array}{l}\mathrm{p}= \\
.992\end{array}$ \\
\hline $\begin{array}{l}\text { Education, } \\
\text { years (mean } \\
\pm \mathrm{SD} \text { ) }\end{array}$ & $13.6 \pm 3.7$ & $\begin{array}{l}13.85 \pm \\
2.64\end{array}$ & $\begin{array}{l}14.9 \pm \\
4.52\end{array}$ & $\begin{array}{l}F= \\
0.756^{(a)}\end{array}$ & $\begin{array}{l}\mathrm{p}= \\
.474\end{array}$ \\
\hline $\begin{array}{l}\text { Age of onset, } \\
\text { years (mean } \\
\pm \text { SD; range) }\end{array}$ & $\begin{array}{l}25.6 \pm 6.9 \\
18-41\end{array}$ & $\begin{array}{l}26.5 \pm 8.8 \\
16-50\end{array}$ & N/A & $\begin{array}{l}t= \\
-0.276^{(b)}\end{array}$ & $\begin{array}{l}\mathrm{p}= \\
.784\end{array}$ \\
\hline $\begin{array}{l}\text { Duration of } \\
\text { illness, years } \\
\text { (mean } \pm \mathrm{SD} \text {; } \\
\text { range) }\end{array}$ & $\begin{array}{l}6.0 \pm 7,9 \\
1-17\end{array}$ & $\begin{array}{l}5.2 \pm 4.3 \\
1-16\end{array}$ & N/A & $\begin{array}{l}\mathrm{t}= \\
-0.297^{(\mathrm{b})}\end{array}$ & $\begin{array}{l}\mathrm{p}= \\
.769\end{array}$ \\
\hline $\begin{array}{l}\text { Number of } \\
\text { lifetime } \\
\text { admissions } \\
\text { (mean; } \\
\text { range) }\end{array}$ & $1.25 ; 0-7$ & $1.25 ; 0-4$ & N/A & $\begin{array}{l}\mathrm{t}= \\
0.000^{(\mathrm{b})}\end{array}$ & $\begin{array}{l}\mathrm{p}= \\
1.000\end{array}$ \\
\hline $\begin{array}{l}\text { Antipsychotics, } \\
\text { CPZE }\end{array}$ & $\begin{array}{l}380.0 \\
(50-1600)\end{array}$ & $\begin{array}{l}160.8 \\
(0-1066)\end{array}$ & $\mathrm{N} / \mathrm{A}$ & $\begin{array}{l}\mathrm{t}= \\
2.226^{(\mathrm{b})}\end{array}$ & $\begin{array}{l}\mathrm{p}= \\
.032 *\end{array}$ \\
\hline $\begin{array}{l}\text { Psychotropic } \\
\text { treatment, } \\
\text { DDD (range) }\end{array}$ & $\begin{array}{l}0.96(0.20- \\
3.50)\end{array}$ & $\begin{array}{l}0.81 \\
(0.00-2.00)\end{array}$ & N/A & $\begin{array}{l}t= \\
0.755^{(b)}\end{array}$ & $\begin{array}{l}\mathrm{p}= \\
.455\end{array}$ \\
\hline $\begin{array}{l}\text { History of } \\
\text { psychosis, n } \\
(\%)\end{array}$ & $20(100 \%)$ & $16(80 \%)$ & N/A & $\begin{array}{l}\chi^{2}= \\
4.444^{(c)}\end{array}$ & $\begin{array}{l}\mathrm{p}= \\
.106\end{array}$ \\
\hline $\begin{array}{l}\text { History of } \\
\text { substance } \\
\text { use, n (\%) }\end{array}$ & $5(25 \%)$ & $7(35 \%)$ & $\mathrm{N} / \mathrm{A}$ & $\begin{array}{l}\chi^{2}= \\
0.476^{(c)}\end{array}$ & $\begin{array}{l}\mathrm{p}= \\
.731\end{array}$ \\
\hline $\begin{array}{l}\text { History of } \\
\text { suicidal } \\
\text { behaviors, n } \\
\text { (\%) }\end{array}$ & $4(20 \%)$ & $4(20 \%)$ & N/A & $\begin{array}{l}\chi^{2}= \\
0.000^{(\mathrm{c})}\end{array}$ & $\begin{array}{l}\mathrm{p}= \\
1.000\end{array}$ \\
\hline
\end{tabular}

BPD - bipolar disorder; CPZE - chlorpromazine equivalents; CTR - controls; DDD - defined daily dose; SCZ - schizophrenia; SD - standard deviation.

(a) ANOVA; ${ }^{\text {(b) }}$ t Student test; ${ }^{\text {(c) }}$ Chi square.

in SCZ $(32.70 \pm 9.95)$. Regarding the vocabulary subtest of WAIS-III, patients with SCZ had lower scores $(40.80 \pm 8.91)$, with statistical significance, than individuals with BPD $(47.65 \pm 6.93 ; \mathrm{p}=.017)$ or healthy controls $(50.95 \pm 6.97 ; \mathrm{p}<.001)$.

Detailed neuropsychological results on SC can be found in Table 2.

\subsection{ToM judgement behavior analysis}

An Independent Samples Kruskal-Wallis $\mathrm{H}$ test revealed no statistically significant effect of group in expected response performance in controls, SCZ and BPD $[\chi 2(2)=3.783, \mathrm{p}=0.151]$.

A statistically significant effect of animation category was found using a Friedman test, affiliative, antagonistic, indifferent, linear $[\chi 2(3)$ $=22.079, \mathrm{p}<0.001]$. Post-hoc tests with Bonferroni correction for multiple comparisons indicated that participants had lower performance for indifferent than affiliative ( $\left.\mathrm{p}_{\text {Bonferroni }}<0.05\right)$, antagonistic $\left(\mathrm{p}_{\text {Bonfer- }}\right.$ roni $=0.05)$ or linear $\left(\mathrm{p}_{\text {Bonferroni }}<0.05\right)$ animations.

Concerning time spent by participants to report the emotional valence of videos, an Independent Samples Kruskal-Wallis $\mathrm{H}$ test of behavioral data showed no significant effect of group $[\chi 2(2)=1.309, p$ $=0.520]$ : controls, SCZ, BPD. There was a statistically significant difference $[\chi 2(3)=26.980, p<0.001]$ in time spent to report decisions on different types of animation: affiliative, antagonistic, indifferent, and linear. Pairwise comparisons were performed with Bonferroni correction for multiple comparisons, showing that participants were quicker to report a decision after affiliative animations than indifferent ( $\mathrm{p}_{\text {Bonferroni }}$ $<0.001$ ) and linear blocks ( $\mathrm{p}_{\text {Bonferroni }}<0.05$ ).

Illustrated ToM judgement behavioral data can be found in Fig. 1.

\subsection{ToM judgment fMRI data}

The ROI voxel-wise statistical group analysis testing the interaction between animation video category and group revealed a significant effect [F(6,171), $\mathrm{q}(\mathrm{FDR})<0.05, \mathrm{p}<0.000531]$ in two groups of contiguous voxels, labelled as Clusters A and B. Cluster A comprises a region belonging to the right supramarginal gyrus (Brodmann area 40), whereas Cluster $B$ includes part of the left posterior superior temporal gyrus (Brodmann area 22) (Lancaster, 2000); these regions are presented in Fig. 2 and described in Table 3. Notably, several voxels of Cluster A and Cluster B revealed significant interaction effect after a stringent Bonferroni correction for multiple comparisons $[\mathrm{F}(6,171), \mathrm{p}$ (Bonf) $<0.05, \mathrm{p}<0.000054]$. Event-related time course analyses of the two clusters are shown in Fig. 2, showing distinct mean percent BOLD signal change for controls, SCZ and BPD, during affiliative, antagonistic, indifferent and linear animations. The plots of the percent signal change provide an intuitive illustration and evidence of the pronounced differences that exist in the dynamics of the BOLD signals of the different groups and conditions. Concerning main group effects, ROI voxel-wise statistical analysis didn't show significant differences $[\mathrm{F}(2,57)$, $\mathrm{q}$ $($ FDR $)>0.05, \mathrm{p}>0.001030$ ] between controls, SCZ and BPD.

Table 2

Social neurocognition evaluation results.

\begin{tabular}{|c|c|c|c|c|c|c|}
\hline \multirow[t]{2}{*}{ TEST } & \multirow{2}{*}{$\begin{array}{l}\text { Schizophrenia (mean } \pm \\
\text { SD) }\end{array}$} & \multirow{2}{*}{$\begin{array}{l}\text { Bipolar Disorder (mean } \pm \\
\text { SD) }\end{array}$} & \multirow{2}{*}{$\begin{array}{l}\text { Controls (mean } \pm \\
\text { SD) }\end{array}$} & \multicolumn{2}{|l|}{ ANOVA } & \multirow[t]{2}{*}{ Tukey Post Hoc Test } \\
\hline & & & & $\mathrm{F}$ & $\mathrm{p}$ & \\
\hline FEIT & $11.95 \pm 2.96$ & $12.30 \pm 2.77$ & $13.75 \pm 2.38$ & 2.467 & 0.094 & \\
\hline SPS & $32.70 \pm 9.95$ & $39.90 \pm 6.09$ & $49.25 \pm 6.90$ & 22.482 & 0.000 & $\begin{array}{l}\mathrm{SCZ}<\mathrm{CTR}(\mathrm{p}<.001) ; \mathrm{BPD}<\mathrm{CTR}(\mathrm{p}=.001) ; \mathrm{SCZ}<\mathrm{BPD} \\
(\mathrm{p}=.014)\end{array}$ \\
\hline SCST & $8.84 \pm 1.03$ & $9.48 \pm 0.80$ & $9.65 \pm 0.84$ & 4.538 & 0.015 & $\mathrm{SCZ}<\mathrm{CTR}(\mathrm{p}=.016)$ \\
\hline AIHQ & $2.53 \pm 0.76$ & $2.27 \pm 0.75$ & $2.47 \pm 0.63$ & 0.689 & 0.506 & \\
\hline RMET & $23.10 \pm 3.32$ & $21.90 \pm 4.74$ & $24.55 \pm 4.19$ & 2.068 & 0.136 & \\
\hline MZQ-PE & $11.65 \pm 3.60$ & $12.95 \pm 3.28$ & $13.10 \pm 3.16$ & 1.130 & 0.330 & \\
\hline $\begin{array}{l}\text { MZQ- } \\
\text { RA }\end{array}$ & $10.25 \pm 3.16$ & $9.75 \pm 2.51$ & $10.70 \pm 2.23$ & 0.638 & 0.532 & \\
\hline TEQ & $43.15 \pm 7.99$ & $46.25 \pm 4.25$ & $49.05 \pm 4.43$ & 5.147 & 0.009 & SCZ < CTR $(\mathrm{p}=.006)$ \\
\hline
\end{tabular}

FEIT - Face Emotion Identification Test; SPS - Social Perception Scale; SCST - Schema Component Sequencing Task - Revised; AIHQ - Ambiguous Intentions Hostility Questionnaire; RMET - Reading the Mind in the Eyes Test; MZQ - Mentalization Questionnaire; MZQ subscales: RS - Refusing self-reflection, EA - Emotional awareness, PE - Psychic equivalence, RA - Regulation of affect;

TEQ - Toronto Empathy Questionnaire; 

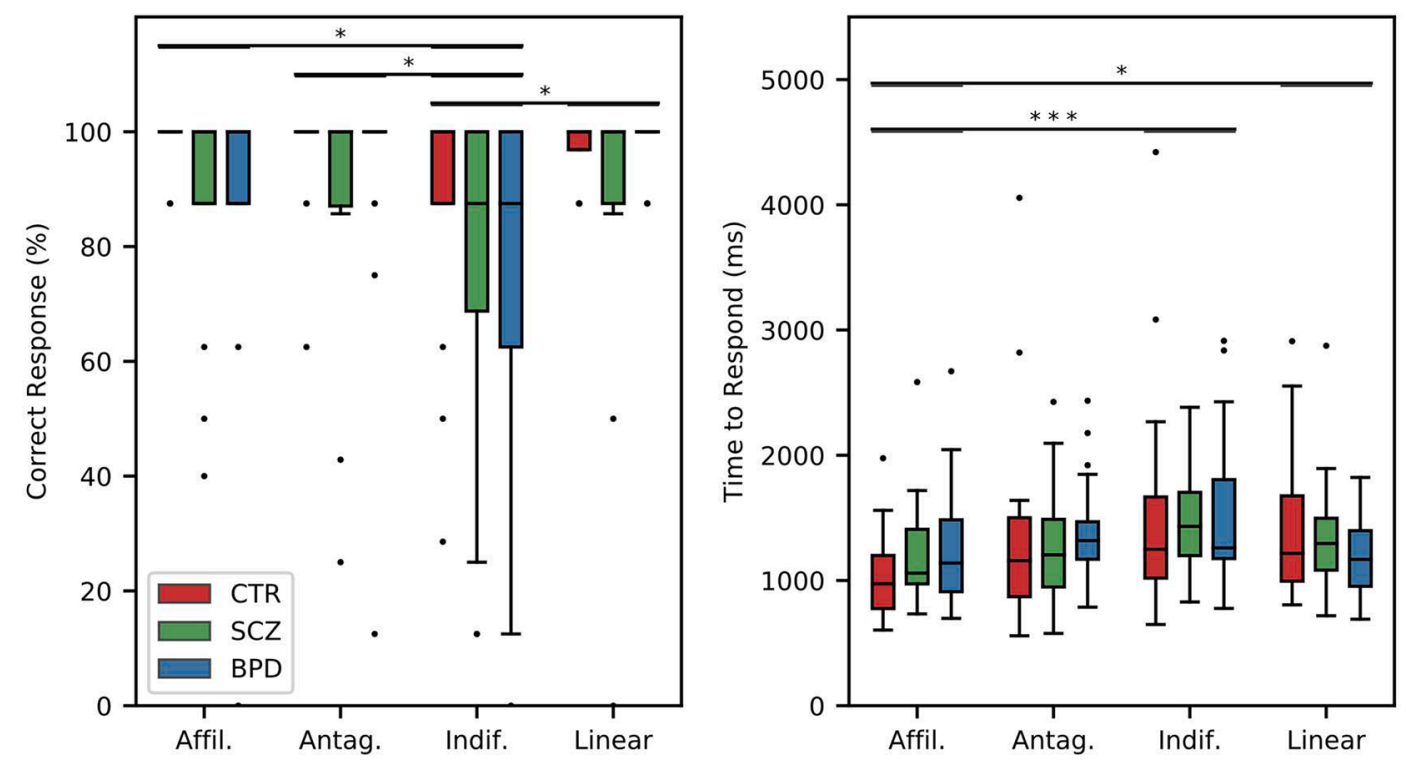

Fig. 1. Left - correct response rate for different stimuli (affiliative, antagonistic, indifferent, linear). Right - mean time spent to report a decision in the decision block. CTR - controls; SCZ - schizophrenia; BPD - bipolar disorder.

Fig. 3 shows the subject-level ROI RFX-GLM beta values of the affiliative, antagonistic, indifferent, and linear predictors in Clusters A (right supramarginal gyrus) and B (left posterior superior temporal gyrus). As expected, the two-way mixed ANOVA, carried out with the ROI RFX-GLM beta values, revealed a statistically significant interaction between the group and the category of animation video in Cluster A [F $(6,171)=4.506, \mathrm{P}_{\text {Bonferroni }}<0.05$, partial $\left.\eta^{2}=0.137\right]$ and Cluster $\mathrm{B}[\mathrm{F}$ $(6,171)=4.669, \mathrm{P}_{\text {Bonferroni }}<0.05$, partial $\left.\eta^{2}=0.141\right]$. In Cluster $\mathrm{A}$, there was a significant simple main effect of group in affiliative $[\mathrm{F}(2,57)$ $=8.533, \mathrm{P}_{\text {Bonferroni }}<0.05$, partial $\left.\eta^{2}=0.230\right]$ and antagonistic $[\mathrm{F}(2,57)$ $=5.764$, $\mathrm{P}_{\text {Bonferroni }}<0.05$, partial $\left.\eta^{2}=0.168\right]$ videos, Pairwise comparisons were performed with Bonferroni correction for multiple comparisons. In both video categories, BPD showed higher beta values than SCZ (affiliative: $\mathrm{P}_{\text {Bonferroni }}<0.05$; antagonistic: $\mathrm{P}_{\text {Bonferroni }}<0.05$ ). In Cluster $\mathrm{B}$, there was no significant simple main effect of group in any category of animation video.

A Pearson's correlation analysis was run to assess the relationship between neurocognition (WAIS vocabulary subtest) or antipsychotics current exposure (chlorpromazine equivalents, CPZE) with the ROI RFXGLM beta values computed in Clusters A and B for CTR, SCZ and BPD groups. Using a tolerant statistical significance threshold ( $\mathrm{p}_{\text {uncorrected }}<$ 0.05), not correcting for multiple comparisons, no significant correlations were found between WAIS vocabulary subtest or CPZE scores and the beta values in clinical groups, across categories of animation videos.

A ROC analysis carried out on the subject-level ROI RFX-GLM beta values showed that single univariate classification by activation in the right supramarginal gyrus provided the best discrimination. Detailed results about the ROC analysis can be found in Table 4. Interestingly, performance was best at discriminating between clinical groups, reaching an AUC of 0.88 for positive stimuli and 0.79 for negative. The ROC analysis of the multivariate classification scores also showed a high AUC at discriminating SCZ and BPD (0.86) using features from the right supramarginal gyrus cluster. The multivariate approach designed with the left posterior superior temporal gyrus features reached an AUC of 0.78 .

\section{Discussion}

The main novelty of this study lies in the discovery of a fundamental neurobiological difference in early social cognition processing between SCZ and BD, using a simple social animation paradigm. We found a critical functional dissociation in implicit ToM in BPD vs. SCZ, with contrasting activation patterns in two core regions with the TPJ complex: the right supramarginal gyrus (SMG) and left posterior superior temporal gyrus (STG). This startling finding was observed in patient groups of short disease duration, with slightly subpar performance in some behavioral and neuropsychological SC measures compared to controls.

Activation of the TPJ, but not of other relevant ToM regions (e.g. PFC), has been found with near-infrared spectroscopy in 7-month-old babies, using ToM visual paradigms, suggesting infants already draw on this region for implicit ToM, similarly to what adults do when reasoning explicitly (Hyde, 2018). Our paradigm, focused on low-level perceptual evaluation, was expected to elicit implicit ToM pathways. These emerge relatively early in both phylogenetic grounds and human neurodevelopment; the right SMG has been known to activate more significantly in somatoperceptive ToM stories, while the more posterior TPJ is associated with ideational story stimuli (Saxe and Powell, 2006).

The nature of differences driving the interaction between group and video categories could relate to right SMG's (which we found to have reduced activity in SCZ and hyperactivity in BPD) role in overcoming the egocentric bias ("overmentalizing”) (Silani, 2013). Conversely, the left posterior STG (underactivated in BPD) is involved in inferring other's intentions and introspective accuracy (Pinkham, 2018). These findings suggest contrasting SC neural dysfunctions in these disorders, as confirmed by our ROC analysis, with robust discriminative power of these regions' activation in distinguishing BPD and SCZ, especially for positive stimuli in the right SMG.

In psychosis, as in autism, there is difficulty setting aside selfknowledge to appreciate another person's subjective world, and it has been so far believed that psychotic and mood conditions were diametric to autism spectrum disorders, associated, respectively, with hyper- or hypofunctioning aspects of SC (Crespi and Badcock, 2008).

Our results bring a new perspective to this view, by showing a critical distinction between SCZ and BPD. This suggests that addressing mentalization as a unidimensional continuum of intercorrelated functions across these disorders could be methodologically fallacious (Langdon and Brock, 2008). ToM dysfunction in SCZ patients could reflect impaired perspective-taking, explaining the egocentric projection of patients' suspicions and biases onto others in ambiguous situations "over-mentalizing", yet failing to grasp the interplay between different perspectives in social interactions - "under-mentalizing" (Langdon and 

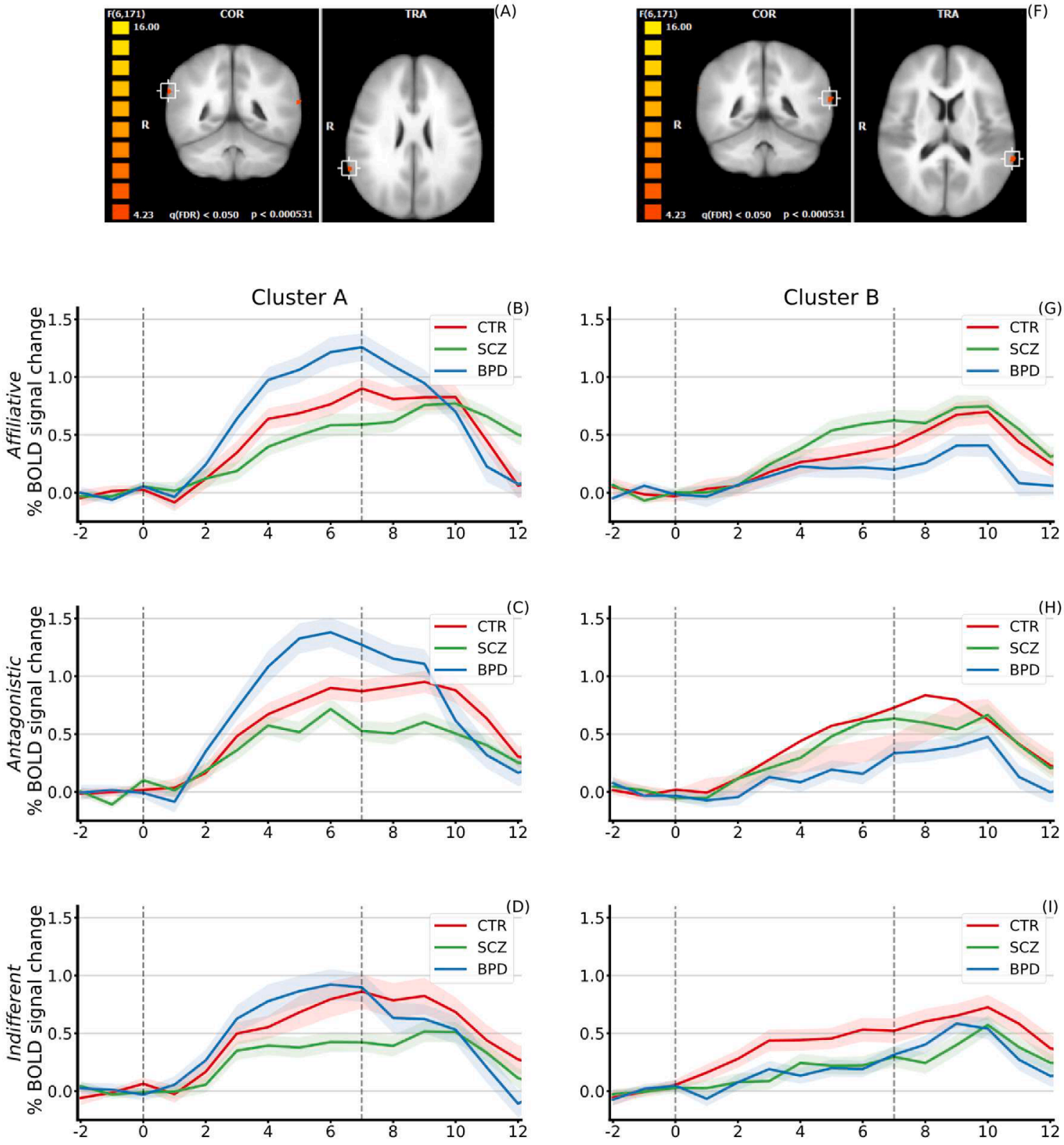

D)
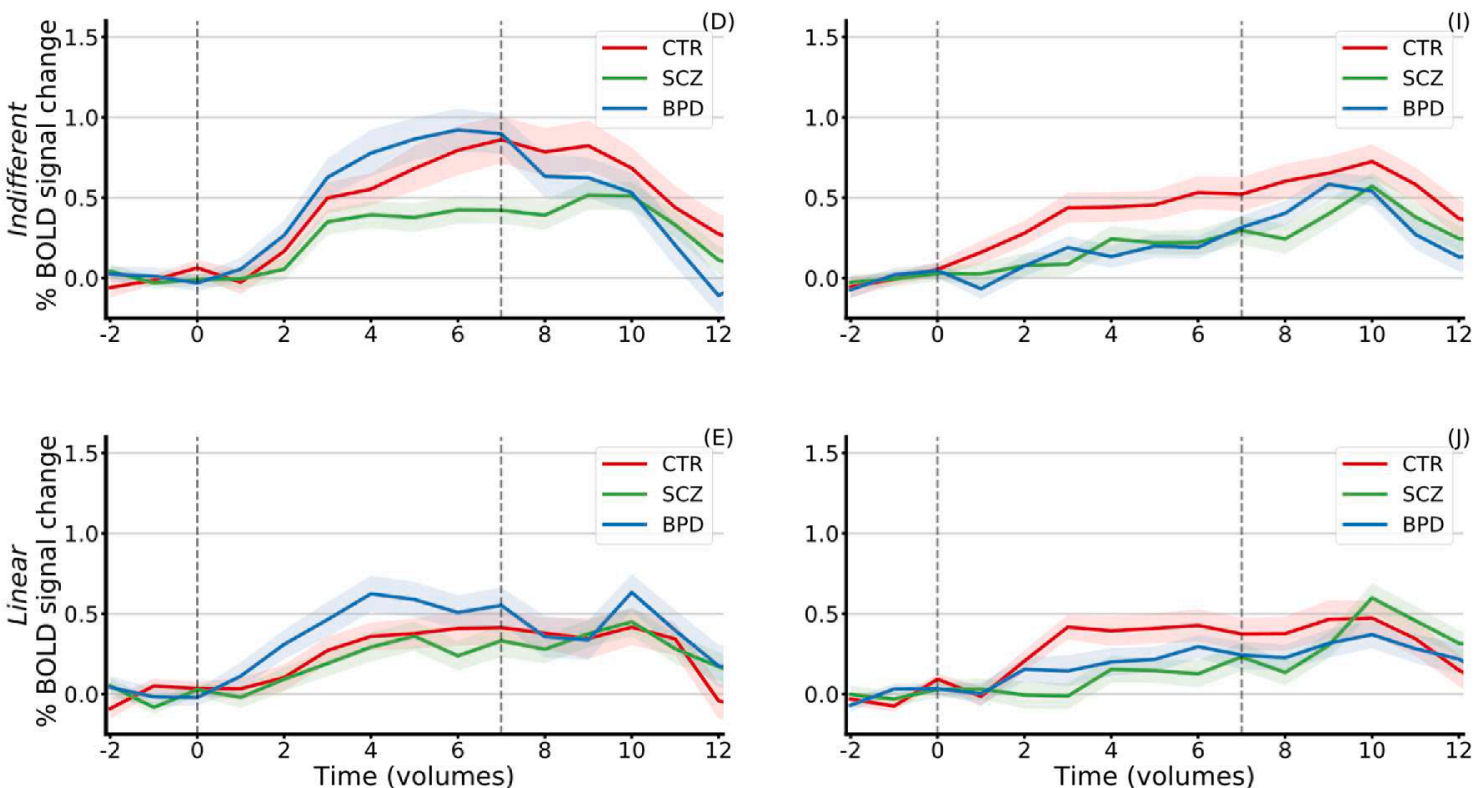
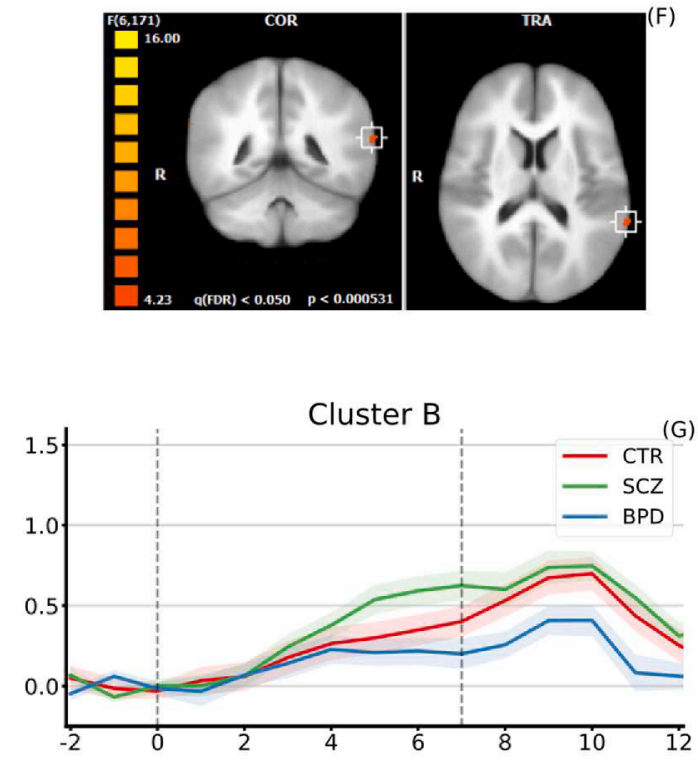

(H) G) 
Table 3

Summary of the ROI voxel-wise statistical group analysis testing the interaction between the category of animation video and group $(\mathrm{q}$ (FDR) $<0.05, \mathrm{P}<$ 0.000531).

\begin{tabular}{llllllll}
\hline Region & No. of Voxels* & \multicolumn{2}{l}{$\begin{array}{l}\text { Peak voxel coordinates } \\
\text { (Talairach) }\end{array}$} & & $\mathrm{F}(6,171)$ & P-value \\
\cline { 3 - 6 } & & $\mathrm{X}$ & $\mathrm{Y}$ & $\mathrm{Z}$ & & \\
\hline Cluster A & $74(6)$ & 63 & -46 & 25 & & 5.564 & 0.000027 \\
Cluster B & $82(4)$ & -66 & -43 & 16 & & 5.286 & 0.000050 \\
\hline
\end{tabular}

*The number of voxels is based on the resolution of the anatomical dataset $1 \times 1 \times 1 \mathrm{~mm}^{3}$.The values in parentheses are the number of significant voxels after Bonferroni correction for multiple comparisons, $\mathrm{p}$ (Bonf) $<0.05$.
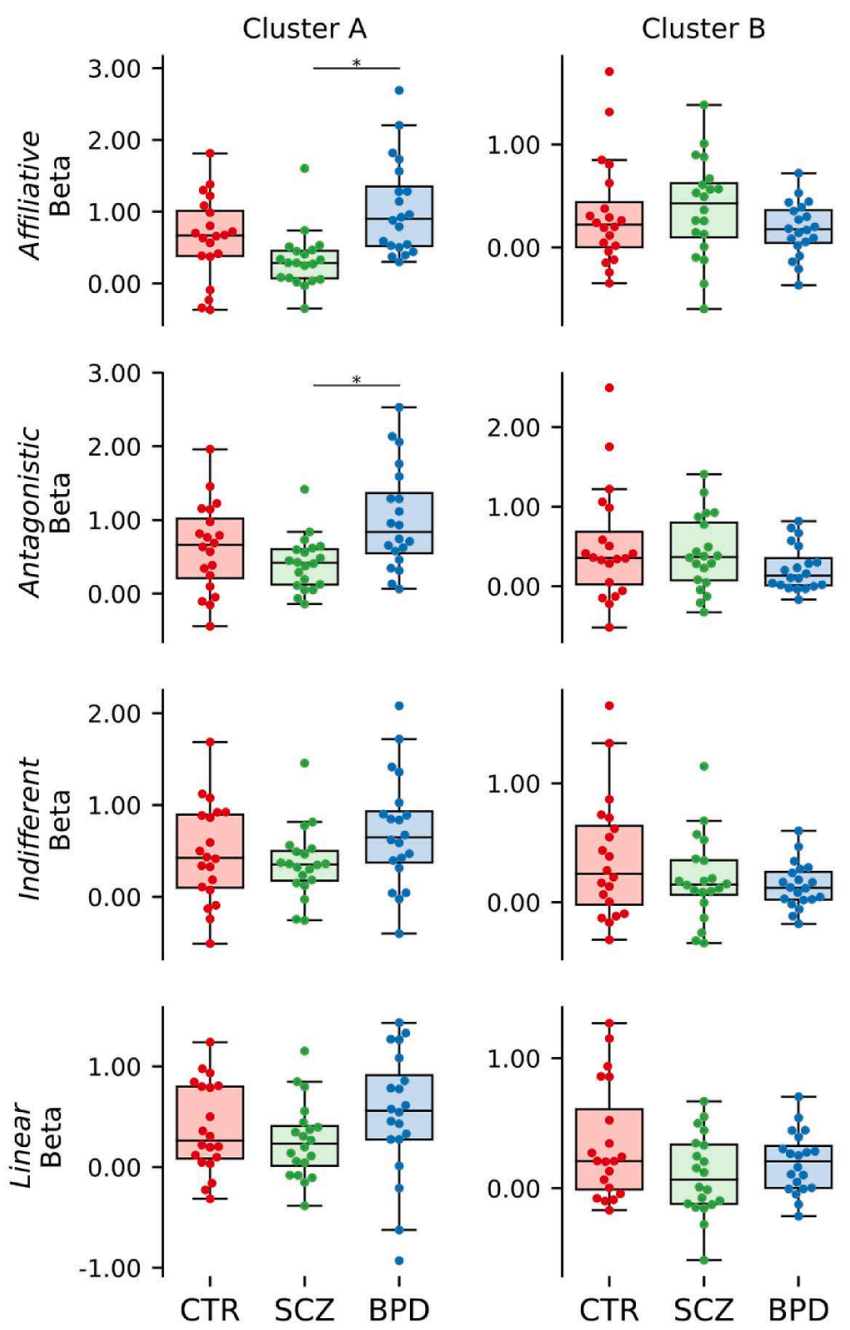

Fig. 3. Result of the ROI RFX-GLM analysis in Cluster A and in Cluster B. Beta values of the affiliative, antagonistic, indifferent, and linear predictors in the Cluster A - right supramarginal gyrus (left) and Cluster B - left posterior superior temporal gyrus (right), for the CTR, SCZ, and BPD groups. CTR - controls; SCZ - schizophrenia; BPD - bipolar disorder.

Brock, 2008). Our study suggests a more fundamental and distinct deficit across conditions occurring even in simple social animations.

Present findings of a dissociation between neural responses of SCZ and BPD in the right SMG - Brodmann area 40 (BA40) - underpin the relevance of this area to ToM processing. An elegant multimodal study, using fMRI and transcranial magnetic stimulation, found that disruption of right BA40 favored one's emotional egocentricity bias - tendency to use the self as a reference point when perceiving the world and emulating others' mental states; right SMG normal activation would be valuable to avoid biased social judgements (Silani, 2013). Independent structural and functional connectivity studies have supported parcellation of the right TPJ, with anterior TPJ, which includes the right SMG, exhibiting robust connectivity with areas relevant to socioemotional processing (e.g. ACC, insula); on the other hand, TPJ posterior areas, including the angular gyrus, have stronger connectivity with the medial PFC (Bzdok, 2013; Mars, 2012; Silani, 2013).

The differential activation of right SMG between BPD and SCZ hereby found is consistent with previous findings regarding degree centrality (DC) - studying the number of instantaneous functional connections between a region and the rest of the brain (Palaniyappan and Liddle, 2014): in SCZ and psychotic BPD patients, using DC from an executive/working-memory fMRI task, DC increase in BPD, compared with SCZ, was found in the right SMG.

The right lateralization of SMG findings is anatomically supported by current knowledge regarding von Economo's neurons (VEN), key functional units to SC and the default-mode network. A study exploring functional connectivity in resting-state of VEN-rich areas found significantly asymmetric preponderance of right vs. left SMG (74 vs. 26\%), something that was not found in other areas, other than the functionally associated right TPJ complex (Cauda, 2013).

In SCZ, where a relative inversion of usual left lateralization of temporal lobe response to language has been reported, it was hypothesized that emotional prosody response could also been inverted, lateralized to the left hemisphere (Woodruff, 1997; Mitchell et al., 2004); such a double inversion was found in a case report after epilepsy neurosurgery (Joseph, 1986). Early neurodevelopmental changes could determine interhemispheric compensation mechanisms. A fMRI study in SCZ and BPD reported left lateralization of response to emotional prosody, along with left SMG hypoactivation in BPD compared to SCZ, and right SMG hypoactivation in BPD compared with controls (Mitchell et al., 2004). Stimuli, unlike our study, were auditory: participants listened to sentences describing happy or sad scenarios.

Morphometric studies pinpointed the SMG as relevant to SCZ and BPD's neuropathology, with volumetric reduction (Amann, 2016; Maggioni, 2017; Nenadic, 2015), predominantly in SCZ. Diffusiontensor imaging also found SMG changes: reduced and augmented fractional anisotropy in SCZ and BPD, respectively, with increased mean diffusivity of grey matter in both (Anderson, 2013). In a recent study with innovative morphometric measures such as gyrification, exploring the present sample, we found increased SMG gyrification in BPD, while in SCZ it was reduced (Madeira, 2020).

A second cluster of differential activation was found in the left posterior STG, with reverse patterns: increased and attenuated response in SCZ and BPD, respectively. This region, home to Wernicke's area, corresponds to the posterior section of Brodmann area 22 (BA22), ventral to the SMG. The superior temporal sulcus (STS) is implicated in understanding others' intentionality, and inferential processing (ToM) has been associated with the BA22 (Lee and Siegle, 2012).

A meta-analysis of functional imaging studies of emotional perception in SCZ reported greater activation in the posterior STG (Taylor, 2012). However, reduced activation of the left STG/BA22 during SC paradigms has been found during acute SCZ episodes (Lee, 2006). In nearly unmedicated first-episode SCZ patients, a diminished contrasting activation of the pSTS has been found when observing intentional vs. random movements of an implicit TdM paradigm (Bliksted, 2019). A recent fMRI study investigated the neural correlates of SC introspective accuracy (IA) in SCZ, measuring activity during a facial emotion recognition task requiring contemporaneous self-assessments; SCZ patients showed reductions in IA-specific neural activity of critical regions for IA, when compared with controls (Pinkham, 2018). Analysis comparing activation in SCZ of IA-specific neural activity vs. a control task reported the highest activation difference in the right STG/BA22 (peak $Z=3.95$ ). Additionally, the left BA22 (peak $Z=3.34$ ) was one of the regions with stronger positive correlation between IA ability and neural activation, in healthy individuals, relative to SCZ. 
Table 4

Classification performance expressed using area under the receiver operating characteristic curve.

\begin{tabular}{|c|c|c|c|c|c|c|c|}
\hline & & \multicolumn{3}{|c|}{$\begin{array}{l}\text { Cluster A } \\
\text { Right Supramarginal Gyrus }\end{array}$} & \multicolumn{3}{|c|}{$\begin{array}{l}\text { Cluster B } \\
\text { Left posterior Superior Temporal Gyrus }\end{array}$} \\
\hline & & $C T R$ vs $S C Z$ & $C T R$ vs $B P D$ & $S C Z$ vs $B P D$ & $C T R$ vs $S C Z$ & $C T R$ vs $B P D$ & $S C Z$ vs $B P D$ \\
\hline \multirow[t]{4}{*}{ Univariate } & Affil. & $0.713(0.090)$ & $0.633(0.089)$ & $0.880(0.054)$ & $0.570(0.093)$ & $0.540(0.093)$ & $0.650(0.091)$ \\
\hline & Antag. & $0.620(0.093)$ & $0.643(0.087)$ & $0.788(0.072)$ & $0.513(0.093)$ & $0.620(0.095)$ & $0.628(0.092)$ \\
\hline & Indif. & $0.570(0.094)$ & $0.598(0.091)$ & $0.703(0.087)$ & $0.595(0.092)$ & $0.608(0.094)$ & $0.545(0.094)$ \\
\hline & Linear & $0.570(0.092)$ & $0.603(0.092)$ & $0.683(0.088)$ & $0.645(0.088)$ & $0.540(0.094)$ & $0.603(0.092)$ \\
\hline Multivariate & & $0.651(0.003)$ & $0.567(0.007)$ & $0.858(0.001)$ & $0.776(0.002)$ & $0.599(0.004)$ & $0.694(0.003)$ \\
\hline
\end{tabular}

In a recent meta-analysis of whole-brain voxel-based morphometry studies in BPD, right STG volume was smaller in some subgroups: adult and type I BPD patients (Lu, 2019). An fMRI study found increased activation of left STS in BPD compared to controls and SCZ; this, along with other activation data during emotion processing in BPD, has been interpreted as an excessively activated emotion-processing system, with heightened significance attributed to emotional stimuli, generating hypervalent affective states (Phillips, 2003; Whalley, 2009).

In general, 'hyper-empathy' in BPD, with a trend towards better 'affective' than 'cognitive' empathy, might equate with known PFC dysfunction and relatively preserved limbic function in BPD (Kim, 2009; Shamay-Tsoory, 2009). Non-surprisingly, ToM impairments have been suggested as a BPD trait marker, related neither with illness years nor medication, or state-related variables such acute phases (Willert, 2015; Bora, 2005).

Despite our case-control study's strict design, namely inclusion/ exclusion criteria and matching relevant variables (age, gender, education), with comparable clinical characteristics (e.g. disease years, substance abuse), some limitations should be discussed. First, its crosssectional nature. Also, sample size precludes some assessments, namely the influence of psychotic symptoms in BPD. While we assessed current medication, its possible effect as a confounder (e.g. antipsychotics exposure) cannot be excluded, although the opposite neural changes documented in BPD and SCZ patients could hardly be attributed to current antipsychotic usage, which was intermediate in BPD, high in SCZ, and absent in controls. Also concerning the specificity of the findings, although patients were matched for years of education, global neurocognitive impairment could have interfered in our findings, despite the modest association known between neurocognition and social cognition (Couture et al., 2006). Nonetheless, as presented, no relevant correlations were found between WAIS vocabulary subtest or CPZE scores and the beta values of fMRI data.

Overall, in this functional neuroimaging study we found a neural response dissociation in BPD vs. SCZ during implicit processing in core ToM regions, with contrasting activation patterns found in right SMG and left posterior STG. This shows a fundamental distinction in social cognition processing occurring at a relatively early level in these conditions and that had so far remained unsuspected. This dissociation, between well-matched patients of two disorders sharing morphometric and genetic features (Ivleva, 2010), sheds light to characteristic neural basis of impaired social cognition in SCZ and BPD, where such deficits could be the most significant predictor of functionality (Couture et al., 2006; Lahera, 2012).

While a perceived feature of psychiatric neuroimaging research has been its relative disconnection from healthcare and its unmet needs (Etkin, 2019), these findings could translate into meaningful gains to clinical practice. Right SMG findings could be investigated as a potential diagnostic biomarker of SCZ vs. BPD, given the robust discriminative power of this region's activation, especially in affiliative animations. Studies focusing on connectivity analysis could bring further detail to the biological processes underlying these findings, and specific disease trajectories occurring in BPD and SCZ (Jimenez, 2019). Developmental synaptic dysfunction has been proposed as a likely mechanism of psychotic symptoms, and aberrant connectivity to the TPJ, namely the
pSTS, has been reported in first-episode SCZ (Dietz, 2020). Models derived from extrinsic and intrinsic connections to the TPJ, personalized for specific dysfunctions found in SCZ and BPD patients, could identify ideal anatomical targets for differential therapeutic neuromodulation (e. g. facilitatory or inhibitory transcranial magnetic stimulation), addressing social handicaps faced by these patients in daily life.

\section{CRediT authorship contribution statement}

Nuno Madeira:Conceptualization, Methodology, Validation, Formal analysis, Investigation, Resources, Data curation, Writing - original draft, Project administration,Ricardo Martins:Conceptualization, Methodology, Software, Validation, Formal analysis, Investigation, Data curation, Writing - original draft, Visualization,João Valente Duarte: Validation, Formal analysis, Investigation, Resources, Data curation, Writing - review \& editing,Gabriel Costa:Investigation, Resources, Writing - review \& editing,António Macedo:Conceptualization, Methodology, Writing - review \& editing, Supervision, Project administration,Miguel Castelo-Branco:Conceptualization, Methodology, Resources, Writing - original draft, Supervision, Project administration, Funding acquisition.

\section{Declaration of Competing Interest}

The authors declare that they have no known competing financial interests or personal relationships that could have appeared to influence the work reported in this paper.

\section{Acknowledgements}

We would like to thank the participants for their involvement in this study. We are also very grateful to: Paula Tavares for the ToM visual animation task; Carlos Ferreira and Sónia Afonso for the help with MRI setup and scanning; Licínio Craveiro for English language reviewing. We acknowledge the anonymous reviewers' insightful comments and improvement suggestions.

\section{Funding}

Financial support for the conduct of this research was granted by the Portuguese Foundation for Science and Technology (FCT), through the projects 'From molecules to man: novel diagnostic imaging tools in neurological and psychiatric disorders' (reference CENTRO-07-ST24FEDER-00205 and BIGDATIMAGE, CENTRO-01-0145-FEDER-000016), 'MEDPERSYST: Synaptic networks and Personalized Medicine Approaches to Understand Neurobehavioral Diseases Across the Lifespan' (reference SAICTPAC/0010/2015, POCI-01-0145-FEDER-016428), PTDC/PSI-GER/1326/2020 and FCT UID/4950/2020. FCT also funded individual grants to JVD (Individual Scientific Employment Stimulus 2017 - CEECIND/00581/2017) and RM (Institutional Call to Scientific Employment Stimulus 2018 - CEECINST/00041/2018). 


\section{Disclosures}

The authors declare no financial disclosures or competing interests.

\section{Appendix A. Supplementary data}

Supplementary data to this article can be found online at https://doi. org/10.1016/j.nicl.2021.102836.

\section{References}

Adolphs, R., 2001. The neurobiology of social cognition. Curr. Opin. Neurobiol. 11 (2), 231-239.

Kern, R.S., Horan, W.P. Definition and Measurement of Neurocognition and Social Cognition, in Neurocognition and social cognition in schizophrenia patients: basic concepts and treatment, in: Roder, V., Medalia, A., Eds. 2010, Karger: Basel Switzerland ; New York. p. xi, 178 p.

Green, M.F., Horan, W.P., 2010. Social cognition in Schizophrenia. Curr. Direct. Psychol, Sci. 19 (4), 243-248.

Premack, D., Woodruff, G., 1978. Does the chimpanzee have a theory of mind? Behav. Brain Sci. 4 (4), 515-629.

Couture, S.M., Penn, D.L., Roberts, D.L., 2006. The functional significance of social cognition in schizophrenia: a review. Schizophr. Bull. 32 (Suppl 1), S44-S63.

Roberts, D.L., Penn, D.L., 2013. Social Cognition in Schizophrenia: From Evidence to Treatment. Oxford University Press, Oxford; New York, p. 436.

Lahera, G., et al., 2012. Social cognition and global functioning in bipolar disorder. J. Nerv. Ment. Dis. 200 (2), 135-141.

Cusi, A.M., et al., 2012. Systematic review of the neural basis of social cognition in patients with mood disorders. J. Psychiatry Neurosci. 37 (3), 154-169.

Rees, E., Owen, M.J., 2020. Translating insights from neuropsychiatric genetics and genomics for precision psychiatry. Genome Med. 12 (1), 43.

Cross-Disorder Group of the Psychiatric Genomics, C, 2013. Identification of risk loc with shared effects on five major psychiatric disorders: a genome-wide analysis. Lancet 381 (9875), 1371-1379.

Craddock, N., O'Donovan, M.C., Owen, M.J., 2009. Psychosis genetics: modeling the relationship between schizophrenia, bipolar disorder, and mixed (or "schizoaffective") psychoses. Schizophr. Bull. 35 (3), 482-490.

Adolphs, R., 2009. The social brain: neural basis of social knowledge. Annu. Rev. Psychol. 60 (1), 693-716.

Schurz, M., et al., 2014. Fractionating theory of mind: a meta-analysis of functional brain imaging studies. Neurosci. Biobehav. Rev. 42, 9-34.

Van Overwalle, F., 2009. Social cognition and the brain: a meta-analysis. Hum. Brain Mapp. 30 (3), 829-858.

Clements, W.A., Perner, J., 1994. Implicit understanding of belief. Cognitive Develop. 9 (4), 377-395.

Apperly, I.A., Butterfill, S.A., 2009. Do humans have two systems to track beliefs and belief-like states? Psychol. Rev. 116 (4), 953-970.

Butterfill, S.A., Apperly, I.A., 2013. How to construct a minimal theory of mind. Mind Language 28 (5), 606-637.

Naughtin, C.K., et al., 2017. Do implicit and explicit belief processing share neural substrates? Hum. Brain Mapp. 38 (9), 4760-4772.

Filmer, H.L., Fox, A., Dux, P.E., 2019. Causal evidence of right temporal parietal junction involvement in implicit Theory of Mind processing. NeuroImage 196, 329-336.

Hyde, D.C., et al., 2018. Functional organization of the temporal-parietal junction for theory of mind in preverbal infants: a near-infrared spectroscopy study. J. Neurosci. 38 (18), 4264-4274.

Bzdok, D., et al., 2013. Characterization of the temporo-parietal junction by combining data-driven parcellation, complementary connectivity analyses, and functional decoding. Neuroimage 81, 381-392.

Mars, R.B., et al., 2012. Connectivity-based subdivisions of the human right "temporoparietal junction area": evidence for different areas participating in different cortical networks. Cereb. Cortex 22 (8), 1894-1903.

Patel, G.H., Sestieri, C., Corbetta, M., 2019. The evolution of the temporoparietal junction and posterior superior temporal sulcus. Cortex 118, 38-50.

Green, M.F., Horan, W.P., Lee, J., 2015. Social cognition in schizophrenia. Nat. Rev. Neurosci. 16 (10), 620-631.

Kronbichler, L., et al., 2017. Abnormal brain activation during theory of mind tasks in schizophrenia: a meta-analysis. Schizophr. Bull. 43 (6), 1240-1250.

Kim, E., et al., 2009. Reduced activation in the mirror neuron system during a virtual social cognition task in euthymic bipolar disorder. Prog. Neuro-Psychopharmacol. Biol. Psychiatry 33 (8), 1409-1416.

Malhi, G.S., et al., 2008. A functional MRI study of Theory of Mind in euthymic bipolar disorder patients. Bipolar Disord 10 (8), 943-956.

Willert, A., et al., 2015. Alterations in neural Theory of Mind processing in euthymic patients with bipolar disorder and unaffected relatives. Bipolar Disord. 17 (8), 880-891.

Lee, J., et al., 2013. Social and nonsocial cognition in bipolar disorder and schizophrenia: relative levels of impairment. Am. J. Psychiatry 170 (3), 334-341.

Espírito-Santo, H., et al., 2017. Preliminary validation of the Portuguese Edinburgh Handedness Inventory in an adult sample. Appl. Neuropsychol.: Adult 24 (3), 275-287.

de Almeida, J.C., et al., Brief Psychiatric Rating Scale (BPRS) versão Ampliada (4.0) Portuguesa: Escala, pontos de ancoragem e manual de administração. 1996.
Kay, S.R., Fiszbein, A., Opler, L.A., 1987. The positive and negative syndrome scale (PANSS) for schizophrenia. Schizophr. Bull. 13 (2), 261-276.

Almeida, S., Gamito, A., Maia, T., 2005. O insight nos doentes esquizofrénicos: instrumentos de avaliação. Psilogos 1 (2), 24-34.

Brissos, S., et al., 2012. The Portuguese version of the Personal and Social Performance Scale (PSP): reliability, validity, and relationship with cognitive measures in hospitalized and community schizophrenia patients. Soc. Psychiatry Psychiatr. Epidemiol. 47 (7), 1077-1086.

Kerr, S.L., Neale, J.M., 1993. Emotion perception in schizophrenia: specific deficit or further evidence of generalized poor performance? J. Abnorm. Psychol. 102 (2), 312-318.

Garcia, S., et al., 2003. Application of the IPT in a Spanish sample: evaluation of the "Social Perception Subprogramme". Int. J. Psychol. Psychol. Ther. 3 (2), 299-310.

Corrigan, P.W., Addis, I.B., 1995. The effects of cognitive complexity on a social sequencing task in schizophrenia. Schizophr. Res. 16 (2), 137-144.

Combs, D.R., et al., 2007. The Ambiguous Intentions Hostility Questionnaire (AIHQ): a new measure for evaluating hostile social-cognitive biases in paranoia. Cogn. Neuropsychiatry 12 (2), 128-143.

Baron-Cohen, S., et al., 2001. The "Reading the Mind in the Eyes" Test revised version: a study with normal adults, and adults with Asperger syndrome or high-functioning autism. J. Child Psychol. Psychiatry 42 (2), 241-251.

Hausberg, M.C., et al., 2012. Is a self-rated instrument appropriate to assess mentalization in patients with mental disorders? Development and first validation of the mentalization questionnaire (MZQ). Psychother Res 22 (6), 699-709.

Spreng, R.N., et al., 2009. The Toronto Empathy Questionnaire: scale development and initial validation of a factor-analytic solution to multiple empathy measures. J. Pers. Assess. 91 (1), 62-71.

Wechsler, D., 2008. WAIS-III: Manual da escala de inteligência de Wechsler para adultosTerceira Edição. CEGOC-TEA, Lisboa.

Fuentes-Durá, I., et al., 2019. WAIS-IV performance in patients with schizophrenia. J. Nerv. Ment. Dis. 207 (6), 467-473.

Bright, P., van der Linde, I., 2020. Comparison of methods for estimating premorbid intelligence. Neuropsychol. Rehabil. 30 (1), 1-14.

Tavares, P., Lawrence, A.D., Barnard, P.J., 2008. Paying attention to social meaning: an FMRI study. Cereb. Cortex 18 (8), 1876-1885.

Tavares, P., Barnard, P.J., Lawrence, A.D., 2011. Emotional complexity and the neural representation of emotion in motion. Soc. Cogn. Affect. Neurosci. 6 (1), 98-108.

Amaro, E., Barker, G.J., 2006. Study design in fMRI: basic principles. Brain Cogn. 60 (3), $220-232$.

Lacadie, C.M., et al., 2008. More accurate Talairach coordinates for neuroimaging using non-linear registration. Neuroimage 42 (2), 717-725.

Lancaster, J.L., et al., 2000. Automated Talairach atlas labels for functional brain mapping. Hum. Brain Mapp. 10 (3), 120-131.

Saxe, R., Powell, L.J., 2006. It's the thought that counts: specific brain regions for one component of theory of mind. Psychol. Sci. 17 (8), 692-699.

Silani, G., et al., 2013. Right supramarginal gyrus is crucial to overcome emotional egocentricity bias in social judgments. J. Neurosci. 33 (39), 15466-15476.

Pinkham, A.E., et al., 2018. Neural correlates of social cognitive introspective accuracy in schizophrenia. Schizophr. Res. 202, 166-172.

Crespi, B., Badcock, C., 2008. Psychosis and autism as diametrical disorders of the social brain. Behav. Brain Sci. 31 (3), 241-261 discussion 261-320.

Langdon, R., Brock, J., 2008. Hypo- or hyper-mentalizing: It all depends upon what one means by "mentalizing". Behav. Brain Sci. 31 (3), 274-275.

Palaniyappan, L., Liddle, P.F., 2014. Diagnostic discontinuity in psychosis: a combined study of cortical gyrification and functional connectivity. Schizophr. Bull. 40 (3), 675-684.

Cauda, F., et al., 2013. Functional anatomy of cortical areas characterized by Von Economo neurons. Brain Struct. Funct. 218 (1), 1-20.

Woodruff, P.W.R., et al., 1997. Auditory hallucinations and the temporal cortical response to speech in schizophrenia: a functional magnetic resonance imaging study. Am. J. Psychiatry 154 (12), 1676-1682.

Mitchell, R.L.C., Elliott, R., Barry, M., Cruttenden, A., Woodruff, P.W.R., 2004. Neural response to emotional prosody in schizophrenia and in bipolar affective disorder. Br. J. Psych. 184 (3), 223-230.

Joseph, R., 1986. Reversal of cerebral dominance for language and emotion in a corpus callosotomy patient. J. Neurol. Neurosurg. Psychiatry 49 (6), 628-634.

Amann, B.L., et al., 2016. Brain structural changes in schizoaffective disorder compared to schizophrenia and bipolar disorder. Acta Psychiatr. Scand. 133 (1), 23-33.

Maggioni, E., et al., 2017. Common and distinct structural features of schizophrenia and bipolar disorder: The European Network on Psychosis, Affective disorders and Cognitive Trajectory (ENPACT) study. PLoS ONE 12 (11), e0188000.

Nenadic, I., et al., 2015. Prefrontal gyrification in psychotic bipolar I disorder vs. schizophrenia. J. Affect. Disord. 185, 104-107.

Anderson, D., et al., 2013. Overlapping and distinct gray and white matter abnormalities in schizophrenia and bipolar I disorder. Bipolar Disord. 15 (6), 680-693.

Madeira, N., et al., 2020. Morphometry and gyrification in bipolar disorder and schizophrenia: a comparative MRI study. NeuroImage: Clinical 26, 102220.

Lee, K.H., Siegle, G.J., 2012. Common and distinct brain networks underlying explicit emotional evaluation: a meta-analytic study. Soc. Cogn. Affect Neurosci. 7 (5), 521-534.

Taylor, S.F., et al., 2012. Meta-analysis of functional neuroimaging studies of emotion perception and experience in schizophrenia. Biol. Psychiatry 71 (2), 136-145.

Lee, K.H., et al., 2006. A functional magnetic resonance imaging study of social cognition in schizophrenia during an acute episode and after recovery. Am. J. Psychiatry 163 (11), 1926-1933. 
Bliksted, V., et al., 2019. Hyper- and hypomentalizing in patients with first-episode schizophrenia: fmri and behavioral studies. Schizophr. Bull. 45 (2), 377-385.

Lu, X., et al., 2019. Structural imaging biomarkers for bipolar disorder: Meta-analyses of whole-brain voxel-based morphometry studies. Depress Anxiety 36 (4), 353-364.

Phillips, M.L., et al., 2003. Neurobiology of emotion perception II: Implications for major psychiatric disorders. Biol. Psychiatry 54 (5), 515-528.

Whalley, H.C., et al., 2009. Functional imaging of emotional memory in bipolar disorder and schizophrenia. Bipolar Disord. 11 (8), 840-856.

Shamay-Tsoory, S., et al., 2009. Neuropsychological evidence of impaired cognitive empathy in euthymic bipolar disorder. J. Neuropsychiatry Clin. Neurosci. 21 (1), 59-67.

Bora, E, et al., 2005. Evidence for theory of mind deficits in euthymic patients with bipolar disorder. Acta Psychiatr. Scand. 112 (2), 110-116.
Ivleva, E.I., et al., 2010. Genetics and intermediate phenotypes of the schizophrenia-bipolar disorder boundary. Neurosci. Biobehav. Rev. 34 (6), 897-921.

Etkin, A., 2019. A reckoning and research agenda for neuroimaging in psychiatry. Am. J. Psychiatry 176 (7), 507-511.

Jimenez, A.M., et al., 2019. Linking resting-state networks and social cognition in schizophrenia and bipolar disorder. Hum. Brain Mapp. 40 (16), 4703-4715.

Dietz, M.J., et al., 2020. Aberrant effective connectivity is associated with positive symptoms in first-episode schizophrenia. Neuroimage Clin. 28, 102444.

Rapoport, J., Giedd, J., Gogtay, N., 2012. Neurodevelopmental model of schizophrenia: update 2012. Mol. Psychiatry 17, 1228-1238. https://doi.org/10.1038/mp.2012.23. 